\title{
HSE Tropative Database: typological issues and programming decisions
}

\author{
Artyom I. Orekhov \\ Higher School of Economics \\ Moscow, Russia \\ artemorekhov99@gmail.com
}

\author{
Roman V. Tarasov \\ Higher School of Economics \\ Moscow, Russia \\ romantarasov2000@yandex.ru
}

\begin{abstract}
This article describes the structure of HSE Tropative Database. This is the first and still the only linguistic resource providing the full information about tropative models of different natural, constructed and extinct languages. It is based on the only paper proposing tropative classification, and data collected by authors themselves. It is also necessary to mention that this one of few databases dedicated to a grammatical derivation in constructed languages (conlangs). All types of conlangs are represented in the database: auxiliary, zonal, artistic, engineered, and also grammarless Emoji. No other typological resources mentioning Emoji as a stand-alone language-like system have been found so far. The article describes theoretical methodology of the database, range of data it provides for each particular language, and its technical features. Every page of a website is described. The site contains two search pages: by single and by multiple parameters. The glossary, explaining the main terms related to tropativity, is also available for users. The main page contains information about the languages processed and about the authors, which is the reason preventing us from providing a link. This project greatly contributes to typology in general, and to constructed language studies. Improvement of functionality and fulfilling the database is our priority and we appreciate any feedback.
\end{abstract}

Key words: tropative, language, linguistic, database, form, predicate

DOI: $10.28995 / 2075-7182-2021-20-1144-1150$

\section{Introduction}

On April, 21, 2021 the HSE Tropative Database was established ${ }^{1}$. This has been the first and yet the only project on typology and classification of tropative constructions. The database works on a Python code, which, along with the corresponding html pages, had been uploaded to PythonAnywhere in order to run the website globally.

Tropative is a lexical derivation having a meaning 'to consider to be $Z$ '. This term was introduced in [Larche 1996], the paper dedicated to Classical Arabic, and used again in [Jacques 2011] about Japhug. The first attempt at typological generalization was made in [Tarasov 2019], which has become the cornerstone for this database.

In Larche's and Jacques' papers, the term 'tropative' was used only for affixes used for creating a verb 'to consider to be $Z$ ' from an adjective $Z$ or a verb 'to be $Z$ '. In both the latest paper and our database, it is used in a wider meaning - verbs or subordinate clauses with a similar meaning are also included. $\mathrm{Z}$ is called characteristic, the one who has an opinion is a subject, and the stimulus is an object. Tropative construction usually looks like ' $\mathrm{X}$ considers $\mathrm{Y}$ to be Z'. For example, English sentence I consider him to be intelligent is an example of analytical tropative.

In [Tarasov 2019], concept of reverse tropative was introduced. It has a meaning of a widespread or traditional opinion about some object and usually looks like ' $\mathrm{Y}$ is considered to be $\mathrm{Z}$ '. The concept of negative tropative, having a meaning of absence of opinion, was introduced in the same paper.

\footnotetext{
${ }^{1}$ https://artemorekhov1999.pythonanywhere.com
} 


\section{Data}

Data for our project had been collected from November, 2018 till December, 2020. 187 languages and 13 dialects had been processed. 4 languages are extinct, 14 are constructed. All language areas are involved, and a language sample can be considered representative. Languages of Russia were the main focus though.

It is necessary to mention that constructed languages are rarely mentioned in typological papers and are usually ignored during such projects. Indeed, the only grammatical typological database for conlangs is [CALS]. No databases describing a conlang typology for a particular derivation were found. However, we realize that this piece of data can be useful.

Firstly, it might be interesting to compare and contrast constructed models with natural ones. Moreover, it will help us find out which types of constructions are selected for languages with different aims (auxiliary, zonal, artistic, etc.). As it was mentioned before, 14 constructed languages were processed. Among them, there are auxiliary (Esperanto, Solresol, etc.), zonal (Interslavic, etc.), artistic (Klingon, etc.) and engineered (Lojban, etc.) languages and Emoji.Our database is probably the first and the only typological resource turning to data from Emoji not as a part of usual language communication, but as a stand-alone system, which is at least similar to a language. Emoji is valuable for us due to lacking grammar, which requires iconicity. It is interesting to explore which lexical strategies are used to express grammatical categories in grammarless systems.

For fulfilling this project, cross-section method was used, which is clearly described in [Tarasov 2019]. It involves requesting translations of several sentences from native speakers of natural languages and users of constructed languages. Each sentence corresponds to a combination of tropative construction types:

1. Direct positive construction: I consider him to be intelligent.

2. Reverse positive construction: He is considered to be intelligent.

3. Direct negative construction: I do not consider him to be intelligent.

4. Reverse negative construction: He is not considered to be intelligent.

A native speaker of each natural language and a user of each constructed language was interviewed in one of the following languages: Russian, English, Persian, Ukrainian or Spanish. The questionnaires were identical.

We understand the drawbacks, disadvantages and restrictions of this method in comparison with grammar descriptions or corpora analysis, or classical elicitation. Firstly, it does not allow us to exclude existence of tropative in a language. Secondly, it gives no opportunity to explore the full range of ways to express tropative. Finally, there is a risk of an informant's mistake.

However, there are some advantages. Grammar descriptions often leave tropative undescribed, especially in case it is analytical in a language. Furthermore, the majority of languages in a sample lack corpora. Thus, there was a choice between grammar and corpora analysis in some languages and elicitation in the others and elicitation in all languages. Our choice of the latter option is explained by the principle of methodological uniformity - it is preferable to compare data collected with the same method.

As far as traditional elicitation is concerned, it is less convenient for informants, and therefore, much less languages would have been processed.

For each language, the following data are registered in the database:

1. Is tropativity detected in a language? Tropativity is a 'conception of a personal meaning about some object' [Tarasov 2019: 1] in general.

Tropativity is detected in 199 of 200 languages. The only language lacking any form of tropativity is Arrernte in which a conception of a personal meaning is absent. 
2. If Answer 1 is affirmative, is semantical tropative detected? Semantical tropative denotes construction with a meaning of tropativity and 'ability to introduce a subject ... explicitly' [Tarasov 2019: 1].

Semantical tropativity is detected in 198 languages. The only tropativity language lacking semantical tropative is Hawaiian, using Class 4 construction. In this language, a tropative subject cannot be stated explicitly:

(1) akamai 'o ia mana'o 'o ia.

intelligent be.3sg $3 \mathrm{sg}$ opinion be.3sg $3 \mathrm{sg}$.

'There is an opinion that (s)he is smart'. [Tarasov 2019: 3]

3. If Answer 2 is affirmative, is syntactical tropative detected? Syntactical tropative is a type of a semantical one, expressed with one finite clause.

Syntactical tropative can be detected in 153 or 154 languages. 44 or 45 languages using semantical tropative lack syntactical tropative and use Class 3 constructions. An example is from Sakhalin Nivkh:

(2) ni k'ımll-dj: if k'oya many-dj.

$1 \mathrm{sg}$ think-pres $3 \mathrm{sg}$ intelligent be-pres.

'I think he is intelligent' [Tarasov 2019: 3]

Discrepancy is explained by the fact that in Klingon example, particle ' $e$ ' can be regarded either as a subordinate clause marker (Okrand's judgement) or as a topic marker (since the verb Har is transitive, and an object is $3^{\text {rd }}$ person):

(3) val ghaH 'e vI-Har.

intelligent $3 \mathrm{sg}$ TOP $1 \mathrm{sgS} .3 \mathrm{O}$-believe.

'I find him/her smart' [Tarasov 2019: 9]

4. If Answer 3 is affirmative, is grammatical tropative detected? Grammatical tropative is a tropative in Larche's sense, i. e. syntactical one with a characteristic incorporated into a verb?

Grammatical tropative can be detected in 15 or 16 languages. 138 or 139 languages using syntactical tropative (including Klingon) lack grammatical tropative and use Class 2 constructions. English is an example of such language: I consider him to be intelligent.

Discrepancy is explained by the fact that in Lojban example, predicate jivsu 'to have an opinion' can be regarded either a monosemic tropative verb or as a copula of a triadic predicate:

(4) mi jinvi lodu'u ra mencre.

1sg TROP TOP 3sg intelligent.

'I consider him/her to be intelligent' [Tarasov, Akifi 2020: 11]

5. If Answer 4 is affirmative:

5.1. What is the form of a tropative affix/clitic/copula?

5.2. Is grammatical tropative monosemic or polysemic? Is it strong or weak? Strong grammatical tropative can be combined with any stem of a particular class, while weak one can be combined just to some stems.

6. If Answer 3 is affirmative:

6.1. Which predicate is used for tropative, is it monosemic or polysemic? 
6.2. Which cases do subject, object and characteristic require?

7. If Answer 2 is affirmative:

7.1. Is reverse tropative construction used? If so, how is it formed? If it is a result of a grammatical passivization or intransitivization, formula pass(T) or intrans(T) is used, with $T$ standing for a predicate of a direct positive tropative.

7.2. If different predicate is used, what is it?

7.3. Which cases do object and characteristic require?

8. If Answer 1 is affirmative, is negative tropative used? If so, how is it formed? If it is a result of a grammatical negation, formula neg(T) is used.

8.1. If different predicate is used, what is it? If different cases are required, what is the difference?

9. If Answer 2 is affirmative, is reverse negative tropative used? If so, how is it formed? If grammatical passivization, intransitivization or negation is applied, the following formulas can be used:

- $\operatorname{neg}(\operatorname{pass}(\mathrm{T}))$ or neg(intrans(T)) if both operations are applied.

- $\operatorname{neg}\left(\mathrm{T}^{\prime}\right)$ with $\mathrm{T}$ ' standing for positive reverse tropative if the system is direct-reverse asymmetric.

- pass(-T) with -T standing for direct negative tropative if the system is positive-negative asymmetric.

9.1. If the system is completely asymmetric, which predicate is used? Which cases does it require?

For example, the Russian language has the following tropativity description:

1. Tropativity is detected. Personal opinion can be expressed by several verbs: dumatj 'think', sčitatj 'count', etc.

2. Semantical tropative is detected. All the mentioned verbs allow an explicitly expressed subject.

3. Syntactical tropative is detected, because the verb sčitatj 'count' is transitive.

4. Grammatical tropative is not detected, so, question 5 is omitted.

5. E.g., predicate sčitatj is used for tropative, which is polysemic with a meaning 'to count'. Subject requires nominative, object requires accusative, and characteristic requires instrumental.

6. Reverse tropative is used, tropative system is direct-reverse symmetric - ' $\mathrm{T}=$ pass $(\mathrm{T})$. Object requires accusative, while characteristic requires instrumental.

7. Negative tropative is used, tropative system is positive-negative symmetric $-\mathrm{T}=\operatorname{neg}(\mathrm{T})$

8. Negative reverse tropative is $\operatorname{neg}(\operatorname{pass}(\mathrm{T}))$

\section{Table analysis}

Once the user sends a request to the website, the program it runs on analyzes the CSV table, searching by the columns. If the user input matches the definition in the database, the entire CSV line is added to a JSON dictionary along with the language name and it's characteristics.

After the search process is done, the JSON dictionary is then presented in a separate browser tab. In order to make it more suitable for users, it is turned into a table. If the user input does not match any parameter from the table, an error page is instead brought up. 


\section{Website structure}

The website consists of the following pages:

1. Main page.

2. Glossary.

3. Search.

4. Advanced search.

Each page is described below.

\subsection{Main page}

The main page tells the main information about the tropative research, which covers the project supervisor (Oleg Volkov), the main thesis, the main methods of collecting data and language processing. Besides that, the page also shows a list of languages which have tropative.

The main page gives links to glossary and two search pages, one with a single parameter and one with multiple parameters.

\subsection{Glossary}

In a glossary, the user can find information about different interpretations of tropative and explanation of the key terms (tropativity, tropativity types, cases and constructions).

\subsection{Search}

Here, the user needs to perform the independent database search through only one parameter. In total, there are 18 search fields to choose from.

1. Language name version 1 - user enters any set of letters; the program then looks through the language names and looks if the user input is included in any language name.

2. Language name version 2 - gives a dropdown list of languages for the user to choose from.

3. Tropative class - asks the user if the language has a detectable tropative class.

4. Semantical tropative - asks the user if the language has a semantical tropative.

5. Syntactical tropative - asks the user if the language has a syntactical tropative.

6. Grammatical tropative - asks the user if the language has a grammatical tropative.

7. Grammatical tropative form - asks the user which grammatical tropative form the language has.

8. Grammatical tropative type - asks the user which grammatical tropative type the language has.

9. Syntactical tropative predicate - asks the user which syntactical tropative predicate the language has.

10. Direct tropative subject case - asks the user which direct tropative subject case the language has.

11. Direct tropative object case - asks the user which direct tropative object case the language has.

12. Direct tropative characteristics case - asks the user which direct tropative object case the language has. 
13. Reverse tropative predicate - asks the user which reverse tropative predicate the language has.

14. Special stem for reverse tropative - asks the user to enter special stem for it's reverse tropative.

15. Reverse tropative object case - asks the user which reverse tropative object case the language has.

16. Reverse tropative characteristics case - asks the user which reverse tropative characteristics case the language has.

17. Negative tropative predicate - asks the user which negative tropative predicate the language has.

18. Negative reverse tropative - asks the user which negative reverse tropative the language has.

The single parameter search has one unique feature. Since the user searches through the database by only one parameter of their choice, that parameter is highlighted in the output table's headers.

\subsection{Advanced search}

This page is based on the single parameter search, but allows the user to choose multiple parameters at once before hitting the "Search" button at the bottom of the page. It also takes out both language search prompts, reducing the number of search fields to 16. Depending on which boxes the user checks, certain parameters will become locked and unaccounted for search. The full query of limiters is listed below.

1. If the tropativity is marked as negative, all other search options are automatically disabled.

2. If the semantical tropativity detection is marked as negative, all other search options with the exception of negative tropative predicate and negative reverse tropative are automatically disabled.

3. If the syntactical tropativity detection is marked as negative, all syntactical, grammatical and direct tropative case options are automatically disabled.

4. If the grammatical tropativity detection is marked as negative, all options that are related to the grammatical tropative are automatically disabled.

5. If the reverse tropativity detection is marked as negative, any options regarding the reverse tropative case are automatically disabled.

\section{Our future work}

Our web application is not flawlessly coded at the moment of this document being written, and it is not devoid of bugs which might appear on some occassions. Locating the bad code lines and improving them is the top priority.

Another problem is the poor website design. For the time being, it has black letters on the white background. In order to make it more pleasant to users, some design tweaks will be implemented in the near future.

Finally, the main database itself might need expansion. The CSV file used for such purpose does not cover the whole extent of tropative languages, and that's why the database gets constantly updated.

\section{Conclusion}

Overall, our database is the first dedicated to tropative. It is important to remember, that prior to [Tarasov 2019] there was no established classification or terminology of tropative studies. This must mean that there might be some theoretic drawbacks. Some modification is also required. However, this project greatly contributes to typology in general, and to constructed language studies. Improvement of functionality and fulfilling the database is our priority and we appreciate any feedback. 


\section{References}

[1] Guillaume Jacques. Applicative and Tropative Derivations. Linguistics of Tibeto-Burman area, 1-13, 2011.

[2] Pierre Larche. Dérivation lexicale et relation passif en arabe classique. (French) [Lexical derivation and passive relation in classic Arabic]. Journal Asiatique, 265, 1905.

[3] Roman Tarasov. Towards a Typology of Tropative. Conference on Typology and Grammar for Young Scholars. Saint-Petersburg, 2019.

[4] Roman Tarasov, Marwa Akifi. Gunešenāsi-ye Teroupātif (Persian). [Typology of Tropative]. Tbilisi, 2020. 